\title{
Early Determination of TAPSE (Tricuspid Annular Plane Systolic Excursion) Improves Early Identification of Pulmonary Thromboembolism and Management in the Female Population Presenting with Undifferentiated Dyspnea to the Emergency Department
}

\author{
Hymavathi Akula ${ }^{1}$ Ashima Sharma ${ }^{1} \quad$ K. Jeethender Jain ${ }^{2}$
}

\footnotetext{
1Department of Emergency Medicine, Nizam's Institute of Medical Sciences, Hyderabad, Telangana, India

2Department of Cardiology, Nizam's Institute of Medical Sciences, Hyderabad, Telangana, India62108112
}

Address for correspondence Dr. Hymavathi Akula, MD, NIMS, Room No 130, First Floor Trauma Block, Hyderabad, 500082, Telangana, India (e-mail: hyma1310@gmail.com).

Ind J Car Dis Wom 2021;6:108-112.

\begin{abstract}
Keywords

- female

- India

- pulmonary

thromboembolism

$-V T E$

Pulmonary embolism (PE) can present to emergency departments with various clinical symptoms. It can be easily missed. Although both sexes are equally susceptible to it, but factors associated with the use of oral contraceptives and hormonal therapy and pregnancy put the female population more at risk theoretically. Apart from gender-based differences, we have observed that early recognition of PE is dependent on many parameters, with varying degrees of accuracy. The gold standard CT pulmonary angiography is logistically impossible in an unstable patient. Estimation of TAPSE values by performing bedside echocardiography is found to be very sensitive and specific for PE. Our study denotes a cutoff point of $1.8 \mathrm{~cm}$ for females with $\mathrm{PE}$ and $1.07 \mathrm{~cm}$ for males with PE
\end{abstract}

\section{Abstract Image}

DOI https://doi.org/ 10.1055/s-0041-1732516
(C) 2021. Women in Cardiology and Related Sciences.

This is an open access article published by Thieme under the terms of the Creative Commons Attribution-NonDerivative-NonCommercial-License, permitting copying and reproduction so long as the original work is given appropriate credit. Contents may not be used for commercial purposes, or adapted, remixed, transformed or built upon. (https://creativecommons.org/licenses/by-nc-nd/4.0/).

Thieme Medical and Scientific Publishers Pvt. Ltd. A-12, 2nd Floor, Sector 2, Noida-201301 UP, India 


\section{Background}

The prevalence of cardiovascular disease, encompassing deep venous thrombosis (DVT) and pulmonary thromboembolism (PTE) has increased alarmingly by nine-old in Asian females. Most of this data is from the Indian subcontinent. ${ }^{1}$ The difference in epidemiology of VTE and pulmonary embolism (PE) between males and females, if present, is very intriguing. Unfortunately, awareness among Indians regarding this potentially life-threatening disease is low. Risk factors like tobacco chewing and chronic obstructive pulmonary disorder (COPD) are common in females from rural India, whereas hypertension, obesity, diabetes mellitus (DM), heart failure, and sepsis are often the comorbid conditions in urban Indian females. The important point is that the presentation is highly varied; hence, it has never been an easy task to diagnose PTE in the absence of strong previous history of DVT or VTE. The right heart strain or failure associated with acute presentation is life-threatening, as it gets further complicated with hypoxia, hypercarbia, hypotension, and acidosis. Point-of-care ultrasound (POCUS) is the term used for bedside ultrasonography or echocardiography done by emergency physicians on arrival to check for signs specific to PTE. ${ }^{2}$ Recently, a major focus of research has shifted on calculation of tricuspid annular plane systolic excursion (TAPSE). The Western literature suggests a cutoff point between 1.4 to 1.8 to rule out PTE. ${ }^{3}$ The sensitivity and specificity of the parameter are assuring. ${ }^{4}$ The gold standard of confirming PTE is CT pulmonary angiogram (CTPA) scan. However, we realize that it is often logistically impossible to shift an unstable patient to the CT room. Hence, bedside evaluation should be given more importance in initiating anticoagulation therapy to prevent propagation of thrombus and plan for thrombolytic therapy.

\section{Aims and Objectives}

Primary aim-To find the clinical and statistical correlation between TAPSE and a positive CTPA scan.

1. Secondary aims -1 . To find the correlation between the commonly used Wells' score and a positive CTPA scan.

2. To find whether D-dimers in Indian female population increases with PTE occurrence and establish the cutoff.

3. To verify the echo parameters other than TAPSE in identifying PTE early (clinical and statistical).
4. To compare the clinical, laboratory, and POCUS information between men and women with positive CTPA reports.

\section{Methodology}

The study was planned as one of the parts of my postgraduate thesis project for MD emergency medicine under the guidance of emergency medicine and cardiology. Ethics committee approval was obtained before starting the project. A sample size of 30 females and equal number of males was determined using an online sample calculator app, with a power of $90 \%$ and $\alpha$ error of 0.05 . The study period was from February 2019 to October 2019. The patients presented to the academic emergency medicine department of Nizam's Institute of Medical Sciences, Hyderabad, India. The consecutive patients who presented to the history with dyspnea, tachypnea and other symptoms of nonspecific chest pain, syncope, and hemoptysis were subjected to ECG investigation. ST elevation or non-ST elevation-acute coronary syndrome (ACS) was ruled out before considering patients for inclusion in the study. The age of patients was above 18 years. There was no predefined upper age limit. However, very elderly patients were considered to be prior suffering with cardiorespiratory illnesses and hence were excluded from the study. No patient with symptoms suggestive of asthma, COPD, restrictive lung disease, left ventricular (LV) dysfunction, and chronic kidney disease (CKD) were included in study. Conditions like valvular heart diseases, especially mitral valve dysfunction, obesity hypoventilation syndrome and chronic liver disease (CLD) were also not considered. The patients were resuscitated with a fluid bolus and norepinephrine infusion if systolic blood pressure (SBP) was found to be below $90 \mathrm{~mm}$ of $\mathrm{Hg}$. All patients were given $\mathrm{O} 2$ at 10 to 15 liters by nonrebreather mask (NRBM) to a target of SPO2 $\geq 95 \%$ earlier during stabilization. Arterial blood gas (ABG) was done to guide the need for temporary continuous positive airway pressure (CPAP) application. A strong suspicion of PTE was based on high scores on original Wells' criteria. Patients who had a score less than 2 were termed as having a low probability of PE. They were excluded from the study.

Blood investigations with complete blood picture (CBP), liver function test (LFT), kidney function tests (KFT), and $\mathrm{D}$-dimers were sent. The value of serum creatinine is obtained and mentioned in the requisition form for CTPA. Patients with serum creatinine more than $2.0 \mathrm{mg} / \mathrm{dl}$ were

Table 1 Comparative analysis of patients' vital signs and Wells' score at the time of presentation to ED

\begin{tabular}{|l|l|l|l|}
\hline Parameter & Females & Males & $p$-Value \\
\hline HR (mean) & 115 & 119 & 0.89 \\
\hline SBP (mean) & 116.47 & 119.06 & 0.88 \\
\hline RR (mean) & 27 & 28.09 & 0.91 \\
\hline SpO2 (mean) & 71.29 & 89.72 & $0.001^{*}$ \\
\hline Nonrecordable BP at admission (number of patients) & 0 & 3 & - \\
\hline High probability on Wells' score (number) & 6 & 11 & $0.12^{*}$ \\
\hline
\end{tabular}

Abbreviations: BP, blood pressure; HR, heart rate; RR, respiratory rate; SBP, systolic blood pressure. 
excluded from the study. POC D-dimers (Abbott triage meter) were done immediately, and emergency physician (EP) performed a bedside echocardiography for the patient along with a cardiology resident. The anticoagulation orders for the patient or the cath laboratory intervention was decided by the cardiologist.

\section{Results}

We received 27 females and 30 males with suggestive symptoms and high probability of PTE on Wells' score during the study period. However, nine females were not included in the final analysis, as they left against medical advice in view of financial constraints before CTPA (which is our final diagnostic criteria in our study). PSPP version 12.0 was used for statistical analysis. A total of 11 females with positive CTPA report were younger $(44.19 \pm 8.56)$ years than those with final negative report of CTPA (49.01 \pm 6.80$)$ years. The 27 CTPA-positive males were older $(42 \pm 2)$ than the three men who were CTPA-negative $(37 \pm 3)$.

Five females were using oral contraceptive pills (OCPs) on prescription, two were known cases of connective tissue disorders, and three had high body mass index (BMI). Among the males, varicose veins were seen in nine subjects, morbid obesity in two, and five of them were chronic smokers. The three most common and statistically significant presentations in females were shortness of breath (SOB), cough and chest pain. SOB was the most common presentation in male patients also; however, the next two common symptoms were orthopnea and lower limb pain (calf pain). The most common signs of PTE in our study for the entire population were tachypnea and tachycardia. - Table 1 compares the vitals on presentation and Wells' score between female and male patients.

Table 2 Crosstabs parameters versus positive CTPA results (Fischer exact test)-gender-based analysis

\begin{tabular}{|l|l|l|}
\hline $\begin{array}{l}\text { Parameter- } \\
\text { symptoms and } \\
\text { signs }\end{array}$ & $\begin{array}{l}\text { Exact sig } \\
\text { (1-tailed) } \\
\text { females }\end{array}$ & $\begin{array}{l}\text { Exact sig } \\
\text { (1-tailed) } \\
\text { males }\end{array}$ \\
\hline SOB & $0.002^{* *}$ & $0.001^{* *}$ \\
\hline Chest pain & $0.060^{\wedge}$ & 0.58 \\
\hline Cough & 0.394 & 0.66 \\
\hline Hemoptysis & 0.598 & - \\
\hline Orthopnea & 0.484 & $0.07^{\wedge}$ \\
\hline Syncope & 0.588 & 0.714 \\
\hline Calf pain & 0.686 & $0.09^{\wedge}$ \\
\hline Lower limb swelling & 0.484 & 0.591 \\
\hline $\begin{array}{l}\text { Tachycardia on } \\
\text { presentation }\end{array}$ & $0.051^{*}$ & $0.007^{* *}$ \\
\hline $\begin{array}{l}\text { Hypoxia on } \\
\text { presentation }\end{array}$ & 0.218 & $0.089^{\wedge}$ \\
\hline $\begin{array}{l}\text { Tachypnea on } \\
\text { presentation }\end{array}$ & 0.668 & $0.053^{*}$ \\
\hline
\end{tabular}

Abbreviations: CTPA, CT pulmonary angiogram; SOB, shortness of breath.
The pretest probability score, symptoms, and signs were subjected to nonparametric analysis to find statistical significance. - Table 2 demonstrates that SOB, chest pain, and tachycardia on presentation were significant findings in the female population, whereas SOB, tachycardia, and tachypnea were significant among male patients suffering with PE, as confirmed by CTPA. Calf pain and hypoxia on presentation were clinically significant presentations in male patients.

ABG was suggestive of hypocarbia and hypoxemia in CTPA-positive cases (LR + 4.0). ECG showed T-wave inversion in V3-V6 chest leads as a clinically significant indicator of positive thrombus on CTPA. FOCUS algorithms consisting of acute right ventricular (RV) dilatation and acute tricuspid regurgitation were noticed in $70 \%$ of confirmed PTE in both females and males. These parameters are highlighted in

\section{- Table 3.}

D-dimers were significantly higher in truly positive females (3878 \pm 810 vs. $1688 \pm 321) \mathrm{ng} / \mathrm{mL}$ and had a highly significant area under curve (AUC) of 0.81 in our study. The comparative levels of D-dimers in men were $3530.82 \pm$ $134 \mathrm{ng} / \mathrm{mL}$ in CTPA-positive males versus $2233 \pm 630 \mathrm{ng} / \mathrm{mL}$ in CTPA-negative males. TAPSE values were significant below a cutoff of 1.8 (AUC 0.88 ) with a decrease in sensitivity of TAPSE if cutoff is decreased to 1.2 (AUC 0.31). - Fig. 1 shows the AUC curve for D-dimers and TAPSE values in female population. Comparatively, TAPSE in CTPA-proven PTE in male population was 1.07 (AUC 0.85) and mean TAPSE values in CTPA-unproven PTE was $2.31 \mathrm{~cm}$.

\section{Discussion}

Indian females are more prone to develop VTE and PTE. The incidence of VTE is higher in men in age groups other than between 45 to 80 years. The young women are more prone to $P E$ due to active estrogen and pregnancy-related states, whereas a longer life expectancy is natural to women past 80 years. The overall age- and sex-adjusted annual incidence of VTE is reported to be 117 cases per 100,000 people (DVT, 48 cases per 100,000; PE, 69 cases per 100,000). ${ }^{5}$ The disease is more common to occur in postpartum situations and in

Table 3 Crosstabs parameters versus positive CTPA results (Fischer exact test)-gender-based analysis

\begin{tabular}{|l|l|l|}
\hline $\begin{array}{l}\text { Parameter- } \\
\text { investigations }\end{array}$ & $\begin{array}{l}\text { Exact sig } \\
\text { (1-tailed) } \\
\text { females }\end{array}$ & $\begin{array}{l}\text { Exact sig } \\
\text { (1-tailed) } \\
\text { males }\end{array}$ \\
\hline S1Q3T3 & 0.686 & 0.149 \\
\hline RBBB & 0.588 & 0.224 \\
\hline T-inversion lateral leads & 0.354 & 0.649 \\
\hline $\begin{array}{l}\text { Metabolic acidosis } \\
\text { (compensated) }\end{array}$ & 0.482 & 0.229 \\
\hline $\begin{array}{l}\text { Moderate hyper lactate- } \\
\text { mia > 5 mmol/l }\end{array}$ & 0.176 & 0.149 \\
\hline D-shaped RV & 0.354 & 0.718 \\
\hline McConnell's sign & 0.588 & 0.277 \\
\hline
\end{tabular}

Abbreviations: CTPA, CT pulmonary angiogram; RBBB, right bundle branch block; RV, right ventricle. 

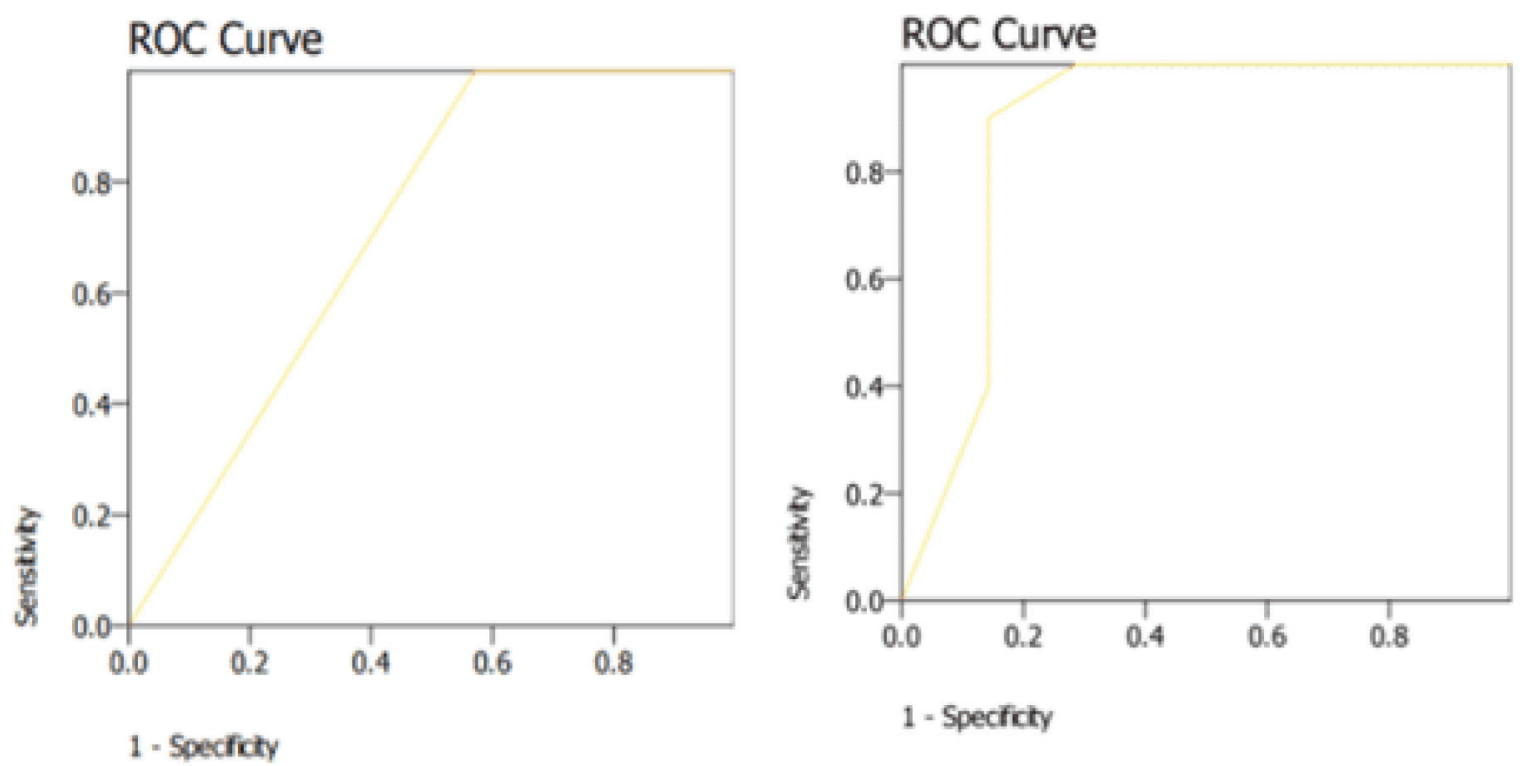

Fig. 1 ROC curves for (a) tricuspid annular plane systolic excursion (TAPSE) and (b) D-dimer.

females with connective tissue disease (CTD), malignancies, or those who are bedridden. A study by Ebadi et al discussed the differences between gender and association with risk factors, clinical symptoms and signs. ${ }^{6}$ They reported SOB as the main clinical symptom in women, whereas SOB and chest pain as two most common symptoms in men afflicted with PTE. Analysis of data from the International Cooperative Pulmonary Embolism Registry (ICOPER), a cohort of 1454 consecutive patients with PE, showed similar prevalence of risk factors in both genders. ${ }^{7}$ The Prospective Investigation of Pulmonary Embolism Diagnosis (PIOPED) trial also studied the gender differences in patients with symptoms and signs very suggestive of PE. Prevalence of PE was higher in men than women (34\% vs. $25 \%$ ) under 50 years, not explained by differences in risk factors. Regarding presenting symptoms and signs, hemoptysis was again more frequent in men $(21 \%$ vs. $10 \% ; p<0.02$ ) as well as leg swelling (36\% vs. $24 \% ; p<0.04){ }^{8}$

Consideration of the probability of PE before testing (that is, pretest probability) avoids unnecessary testing and is critical to the interpretation of results. The Geneva and Wells' rules are among the most commonly cited clinical probability scores. ${ }^{9}$ In a study by Modi et al, performance of Wells' score was found to be clinically accurate and statistically very significant. The patients with DVT had higher scores (2 median, 1-5 interquartile range [IQR]) than those without DVT (1 median, 1-3 IQR). In low-risk patients (scores < 1), Wells' scoring was able to rule out the possibility of DVT with a sensitivity of $100 \%$ and negative predictive value (NPV) of $100 \%$, while in moderate-to-high risk patients (scores $\geq 2$ ), it was able to predict DVT with a specificity of $90 \% .^{10}$ Investigations such as ECG and ABG also have certain pitfalls in correct diagnosis. ECG was found to be neither sensitive nor specific in a review by Oullette et al. ${ }^{11}$ Stein et al had concluded that a normal ECG can be seen in $30 \%$ of patients with PE, whereas the classic S1Q3T3 occurs in only $20 \%$ of patients with angiographically proven PE and has a sensitivity and specificity of 54\% and $62 \%$, respectively. ${ }^{12}$ The most common ECG change is
T-wave inversion in the precordial leads, which is found in 68\% of patients with PE. Sinus tachycardia is the most frequent rhythm seen in patients with PE, occurring in 36\%. ${ }^{13}$ Right bundle branch block (RBBB), P pulmonale, right axis deviation, and new-onset atrial fibrillation are other ECG findings in PE.

In our study, the information obtained from the ABG sample showed hypoxemia and hyperlactatemia in patients with confirmed PE. PIOPED investigators had suggested the unexplained presence of hypoxemia or any sudden change in the A-a gradient increases the likelihood of PE. In patients with normal lungs, PIOPED found that a PCO2 greater than $36 \mathrm{~mm}$ $\mathrm{Hg}$ and a normal alveolar arterial oxygen (A-a) gradient had a $98 \%$ NPV for $\mathrm{PE}^{14}$ Cvitanic and Marino found that $98 \%$ of patients had either an increased A-a gradient or hypocapnia. ${ }^{15}$ The ABG and A-a gradient may be normal in 5 to $35 \%$ of patients with PE and no prior cardiopulmonary disease. ${ }^{16}$

D-dimer estimation had a very significant impact in our study on the positive PE cases. It is a sensitive but not a specific diagnostic test. However, in a recent observational study of 2017 in patients with suspected PE, values below $1000 \mathrm{ng} / \mathrm{mL}$ in patients with a Wells' score less than 2 and $500 \mathrm{ng} / \mathrm{mL}$ in patients with score 2 to 6 could safely rule out PE. Nn further diagnostic testing was advised. ${ }^{17} \mathrm{D}$-dimer concentrations increase with age, and specificity can be improved with an age adjusted cutoff value. An observational study of 3346 patients evaluated an age adjusted D-dimer $(500 \mu \mathrm{g} / \mathrm{L}$ cutoff for patients $\leq 50$ or age $\times 10 \mu \mathrm{g} / \mathrm{L}$ for patients $>50$ years), whereby patients with a negative $\mathrm{D}$-dimer and an unlikely (Wells) or nonhigh (revised Geneva) clinical probability did not undergo diagnostic imaging. ${ }^{18}$ Age-adjusted D-dimer approach increased the yield of true negatives to $30 \%$, even without diagnostic imaging.

A study by Daley et al suggested that FOCUS performed by EPs may be of increased diagnostic utility in patients with tachycardia or hypotension. ${ }^{19}$ As not all patients have these clinical signs, a need for a new diagnostic parameter was considered, preferably noninvasive, as used in critical emergency scenarios. 
Our study is unique in identifying the cutoff value of TAPSE as $1.8 \mathrm{~cm}$ in female patients and $1.5 \mathrm{~cm}$ in male population affected by PE. Daley et al suggested a cutoff of $2.0 \mathrm{~cm}$ may be more appropriate for the diagnosis of PE in the ED, as using a higher cutoff value improves sensitivity of TAPSE for PE. ${ }^{19}$ Ueti et al observed that TAPSE approximates RV ejection fraction with $80 \%$ sensitivity and $75 \%$ specificity ${ }^{20}$.Morita et al validated that the midesophageal 4-chamber view of transesophageal echocardiography (TEE) as a good correlate to RV systolic function, as reflected by RV fractional area change. ${ }^{21}$

Based on our observations, we recommend the use of TAPSE in emergency departments to enhance the diagnostic possibility of PTE in patients presenting with undifferentiated dyspnea.

\section{Limitations}

TAPSE measures the longitudinal excursion of the tricuspid annulus in one dimension, ignoring the transverse contribution of the leaflets' movement to the RV ejection fraction. Patients with preexisting RV diseases like cor pulmonale or cardiomyopathy may not show accurate TAPSE values. The numerical value of TAPSE may be affected by the angle of the M-mode cursor over the tricuspid annulus. The sample size of our study is very small. A decreased TAPSE is not specific to PE. It may also result from pulmonary hypertension and congestive heart failure; therefore, it is very important to follow the inclusion criteria strictly for similar studies.

\section{Audio}

Audio file for this article is available at https://doi. org/10.1055/s-0041-1732516.

\section{Conclusion}

TAPSE can prove as a modality for early identification of PTE and subsequent early institution of medical therapy. This can save many young lives lost to PTE among young females in our country. A strong index of suspicion should be kept.

We are continuing our research further prospectively as and when we receive a female patient who fulfills the inclusion criteria.

\section{External Funding}

Nil.

\section{Graphical Abstract}

\section{Conflict of Interests}

None declared.

\section{References}

1 Kamerkar DR, John MJ, Desai SC, Dsilva LC, Joglekar SJ. Arrive: a retrospective registry of Indian patients with venous thromboembolism. Indian J Crit Care Med 2016;20(3):150-158

2 Lahham S, Fox JC, Thompson M, et al. Tricuspid annular plane of systolic excursion to prognosticate acute pulmonary symptomatic embolism (TAPSEPAPSE study. J Ultrasound Med 2019;38(3):695-702

3 Rydman R, Söderberg M, Larsen F, Caidahl K, Alam M. Echocardiographic evaluation of right ventricular function in patients with acute pulmonary embolism: a study using tricuspid annular motion. Echocardiography 2010;27(3):286-293

4 Lobo JL, Holley A, Tapson V, et al; PROTECT and RIETE investigators. Prognostic significance of tricuspid annular displacement in normotensive patients with acute symptomatic pulmonary embolism. J Thromb Haemost 2014;12(7):1020-1027

5 Silverstein MD, Heit JA, Mohr DN, Petterson TM, O'Fallon WM, Melton LJ, III. Trends in the incidence of deep vein thrombosis and pulmonary embolism: a 25-year population-based study. Arch Intern Med 1998;158(6):585-593

6 Robert-Ebadi H, Le Gal G, Carrier M, et al. Differences in clinical presentation of pulmonary embolism in women and men. J Thromb Haemost 2010;8(4):693-698

7 Goldhaber SZ, Visani L, De Rosa M. Acute pulmonary embolism: clinical outcomes in the International Cooperative Pulmonary Embolism Registry (ICOPER) Lancet 1999;353(9162): 1386-1389

8 Quinn DA, Thompson BT, Terrin ML, et al. A prospective investigation of pulmonary embolism in women and men. JAMA 1992;268(13):1689-1696

9 Lucassen W, Geersing GJ, Erkens PM, et al. Clinical decision rules for excluding pulmonary embolism: a meta-analysis. Ann Intern Med 2011;155(7):448-460

10 Modi S, Deisler R, Gozel K, et al. Wells criteria for DVT is a reliable clinical tool to assess the risk of deep venous thrombosis in trauma patients. World J Emerg Surg 2016;11:24

11 Ouellette DW, Patocka C. Pulmonary embolism. Emerg Med Clin North Am 2012;30(2):329-375, viii

12 Stein PD, Terrin ML, Hales CA, et al. Clinical, laboratory, roentgenographic, and electrocardiographic findings in patients with acute pulmonary embolism and no pre-existing cardiac or pulmonary disease. Chest 1991;100(3):598-603

13 Ferrari E, Imbert A, Chevalier T, Mihoubi A, Morand P, Baudouy M. The ECG in pulmonary embolism. Predictive value of negative $T$ waves in precordial leads-80 case reports. Chest 1997;111(3):537-543

14 Kline JA, Runyon, MS, Pulmonary embolism and deep venous thrombosis. In: Marx JA, Hockenberger RS, Walls RM, eds. Rosen's Emergency Medicine Concepts and Clinical Practice, Vol. 1. 7th ed. Philadelphia: Mosby; 2009 1124-36

15 Cvitanic O, Marino PL. Improved use of arterial blood gas analysis in suspected pulmonary embolism. Chest 1989;95(1):48-51

16 Stein PD, Goldhaber SZ, Henry JW. Alveolar-arterial oxygen gradient in the assessment of acute pulmonary embolism. Chest 1995;107(1):139-143

17 Kearon C, de Wit K, Parpia S, et al. PEGeD Study Investigators. Diagnosis of pulmonary embolism with d-Dimer adjusted to clinical probability. N Engl J Med 2019;381(22):2125-2134

18 Righini M, Van Es J, Den Exter PL, et al. Age-adjusted D-dimer cutoff levels to rule out pulmonary embolism: the ADJUST-PE study. JAMA 2014;311(11):1117-1124

19 Daley J, Grotberg J, Pare J, et al. Emergency physician performed tricuspid annular plane systolic excursion in the evaluation of suspected pulmonary embolism. Am J Emerg Med 2017;35(1):106-111

20 Ueti OM, Camargo EE, Ueti Ade. de Lima-Filho EC, Nogueira EA. Assessment of right ventricular function with Doppler echocardiographic indices derived from tricuspid annular motion: comparison with radionuclide angiography. Heart 2002;88(3):244-248

21 Morita Y, Nomoto K, Fischer GW. Modified tricuspid annular plane systolic excursion using transesophageal echocardiography for assessment of right ventricular function. J Cardiothorac Vasc Anesth 2016;30(1):122-126 